\title{
The Diagnostic Value of Galactomannan Testing in Bronchoalveolar Lavage Fluid on the Diagnosis of Pulmonary Aspergillosis in Patients with Chronic Respiratory Diseases
}

\author{
Hao Lin", Yuan yuan Niu\#, Wei Shui", Qian Gao, Xin Zhang and Chang-ran Zhang* \\ Department of General Practice, The East Division of the First Affiliated Hospital of Sun Yat-sen University, Guangzhou, \\ Guangdong, China
}

\#These authors contributed equally to this work

*Corresponding author: Chang-ran Zhang, The East Division of the First Affiliated Hospital of Sun Yat-sen University, Guangzhou, 510700, China

\section{ARTICLE INFO}

Received: 幽 October 07, 2021

Published: 幽 October 21, 2021

Citation: Hao Lin, Yuan yuan Niu, Wei Shui, Qian Gao, Xin Zhang and Chang-ran Zhang. The Diagnostic Value of Galactomannan Testing in Bronchoalveolar Lavage Fluid on the Diagnosis of Pulmonary Aspergillosis in Patients with Chronic Respiratory Diseases. Biomed J Sci \& Tech Res 39(3)-2021. BJSTR. MS.ID.006312.

Keywords: Bronchoalveolar Lavage Fluid; Galactomannan Testing; Pulmonary Aspergillosis; Chronicrespiratory Diseases; Diagnostic Value

Abbreviations: CRD: Chronic Respiratory Diseases; GM: Galactomannan; BALF: Bronchoalveolar Lavage Fluid; NPV: Negative Predictive Value; PPV: Positive Predictive Value

\section{ABSTRACT}

Background: To evaluate the diagnostic value of galactomannan testing in bronchoalveolar lavage fluid on the diagnosis of pulmonary aspergillosis in patients with chronic respiratory diseases.

Methods: Bronchoalveolar lavage fluid (BALF) was collected from 100 patients with suspected clinical pulmonary Aspergillus infections by means of bronchoscopy. BALF GM test and serum GM test were compared.

Results: 4 patients with incomplete data and follow-up loss were excluded, and a total of 96 patients were included in this study, among which 46 cases were taken as the case group and 50 cases were taken as the control group. The GM level in BALF in case group was significantly higher than that in control group $(\mathrm{p}<0.05)$, and there was no statistically significant difference in serum GM level between case group and control group. When BALF GM testing was positive with cut-off value $>0.5$, the sensitivity, specificity, positive predictive value and negative predictive value in BALF GM testing were $80.4 \%, 48.0 \%, 58.7 \%$ and $72.7 \%$, respectively. Area under ROC curve 0.779 . When BALF GM value 0.96 was taken as the cut-off value, the Youden index reached the maximum, and the sensitivity and specificity were $67.2 \%, 82.0 \%$ respectively, $(p=0.01)$. When serum GM testing was positive with $>0.5$, the sensitivity, specificity, positive predictive value, and negative predictive value in serum GM test were $16.0 \%, 100 \%, 100 \%$ and $22.2 \%$, respectively. Area under ROC curve 0.638 . When serum GM value 0.18 was taken as the cut-off value, the Youden index reached the maximum, and the sensitivity and specificity were $47.8 \%, 83.3 \%$ respectively $(\mathrm{p}=0.255)$.

Conclusion: The diagnostic value of GM detection in BALF is better than in serum for diagnosis of pulmonary aspergillosis in patients with chronic respiratory diseases. BALF GM test is more meaningful in the diagnosis of pulmonary aspergillosis. The best cut-off value of BALF GM testing is 0.96 .

\section{Introduction}

According to the definition of chronic respiratory diseases (CRD) by the World Health Organization, CRD is a group of diseases that affect the airways and other structures of the lungs, the most common include COPD, bronchial asthma, bronchiectasis, etc. [1]. In-depth studies in recent years have found that pulmonary aspergillosis can also occur in patients with chronic respiratory diseases (CRD) [2,3]. As delayed treatment of pulmonary 
aspergillosis always leads to high mortality rate, early recognition of CRD with pulmonary aspergillosis is extremely important. Galactomannan (GM) is a thermally stable polysaccharide on the cell wall of aspergillus filaments, which is released into the blood from the tip of the mycelium during aspergillus growth [2]. GM can be detected in the blood in the early stages of infection. Nevertheless, various factors have been found in clinical practice to cause false positives and false negatives in galactomannan testing. Bronchoalveolar lavage fluid (BALF) can be applied to detect pathogens on lung lesions in the early stage of aspergillus infection.

Although BALF has been recommended for GM testing by domestic and foreign guidelines, there is no unified standard for BALF-GM testing cut-off value [2,3]. In this study, bronchoalveolar lavage fluid (BALF) was collected from 100 patients with suspected clinical pulmonary Aspergillus infections by means of bronchoscopy. BALF GM test and serum GM test were compared to assess the diagnostic value of galactomannan testing in bronchoalveolar lavage fluid on the diagnosis of pulmonary aspergillosis in patients with chronic respiratory diseases.

\section{Patients and Methods}

\section{Patient Selection}

Between June 2019 and December 2019,100 patients with suspected clinical pulmonary aspergillus infections from three different hospitals (50 patients from the Guangzhou Thoracic Hospital,45 patients from the Guangdong Province People's Hospital, and 5 patients from the First Affiliated Hospital of Sun Yat-Sen University, respectively) were enrolled in this retrospective analysis. They all suffered from chronic respiratory diseases include COPD, bronchial asthma, bronchiectasis, etc. Data of all patients were collected, including age, sex, smoking history, past medical history and medication history, length of stay, laboratory tests, chest imaging examination, pathogen examination, lung pathology and bronchoscopy results. Serum and BALF GM tests were performed during their hospitalization. Factors that might cause false positives in the GM test such as piperacillin/tazobactam were excluded. Hematological malignancies, hematopoietic stem cell transplantation, solid organ transplantation, HIV infection, and patients with incomplete clinical data were excluded from the study.

\section{Statistical Analysis}

a. Koimogorov-smirnov test (K-S test) was used by SPSS 25 software to determine whether the target variables were normally distributed. If the measurement data conformed to normal distribution at the same time, it was represented by mean \pm standard deviation $(\mathrm{X} \pm \mathrm{s})$. For the measurement data that did not conform to normal distribution, it was represented by M(P25-P75). The counting data was expressed by percentage or constituent ratio. Independent sample $\mathrm{T}$ test was used for BALF GM values of the case group and the control group, paired sample T test was used for BALF GM values and serum GM values of the case group, non-parametric rank-sum test and Mann-Whitney $U$ test were used for samples that did not conform to normal distribution.

b. SPSS 25 software was used to draw ROC curves of the diagnostic efficacy of BALF and serum GM test in the case group and the control group, and the optimal cut-off value of BALF and serum GM test for pulmonary aspergillosis was calculated.

c. The data of baseline features, clinical features and imaging examination of the subjects were analyzed with the independent sample $\mathrm{T}$ test or chi-square test for normal distribution, and non-parametric rank-sum test for nonnormal distribution. The differences were considered to be statistically significant when $\mathrm{p}<0.05$.

d. According to several guidelines, the cut-off value of GM was between 0.5 and 1.5 , and the cut-off value of $0.5,0.8,0.9,1.0$, 1.2 and 1.5 have been reported in many guidelines and metaanalyses. Sen, spe, positive predictive value (PPV), and negative predictive value (NPV) of BALF GM were calculated.

\section{Results}

Patient characteristics and data.4 patients with incomplete data and follow-up loss were excluded, and a total of 96 patients were included in this study. According to the diagnosis standards of IDSA (2016) [2], 43 patients were diagnosed by pathological data (proven diagnosis), and 3 cases were diagnosed by radiology, etiology, and other clinical examinations (probable diagnosis). Both of them were included in the case group. The control group included 6 cases of possible pulmonary aspergillosis and 44 cases of nonpulmonary aspergillosis. Clinical data of patients were collected (Table 1). The most common clinical symptoms in the case group were cough (41cases,89.1\%), hemoptysis (30 cases,65.2\%) and expectoration (27 cases,58.7\%). Whereas in the control group were cough (36 cases,72.0\%), expectoration (27 cases,54.0\%) and fever (16 cases,32.0\%). The clinical symptoms of hemoptysis and cough were statistically different between the two groups.

The imaging findings of patients in the two groups included nodular shadow, patchy shadow, consolidation shadow, air crescent sign, cavity and aspergillus balls. Nodular shadow (27 cases,58.7\%) and cavity (22 cases,47.8\%) were dominant in the case group, while patchy shadow (14 cases,28.0\%) and nodular shadow (13 cases,26.0\%) were dominant in the control group. The imaging manifestations of nodular shadow, cavity and aspergillus bulb were statistically different between the two groups. Microbiological 
examination results. In the case group there were 21 cases (45.7\%) of positive aspergillus in BALF culture and 3 cases $(6.50 \%)$ of positive aspergillus in BALF smear microscopy. The serum GM value was $0.18(0.12-0.34)$ in the case group and $0.12(0.07-0.21)$ in the control group, showing no statistical difference. BALF GM value was $1.93(0.61-5.78)$ in the case group and 0.51(0.25-0.82) in the control group, $\mathrm{Z}$ value $=-4.709$. BALF GM value in the case group was higher than that in the control group, $\mathrm{P}<0.05$ (Table 2).

Table 1: Baseline characteristics of patients in case group and control group $(\%)$.

\begin{tabular}{|c|c|c|c|c|}
\hline Features & Case group(\%) & Control group(\%) & $\chi 2 /$ tvalue & $P$ value \\
\hline Age (year) & $52.32 \pm 12.83$ & $58.89 \pm 14.42$ & 2.361 & $0.020^{*}$ \\
\hline Gender (male, percentage) & $38(82.61)$ & $31(62.0)$ & 5.034 & $0.025^{*}$ \\
\hline \multicolumn{5}{|l|}{ Underlying diseases } \\
\hline Bronchiectasia & $27(58.7)$ & $6(12.0)$ & 23.158 & $0.001^{*}$ \\
\hline Pulmonary tuberculosis & $38(82.6)$ & $10(20.0)$ & 40.537 & $0.001^{*}$ \\
\hline Bronchial asthma & $1(2.2)$ & 0 & - & 0.479 \\
\hline COPD & $20(43.5)$ & $11(22.0)$ & 5.055 & $0.025^{*}$ \\
\hline ILD & $0(0.0)$ & $2(4.0)$ & - & 0.496 \\
\hline Pleural effusion & $0(0.0)$ & $6(12.0)$ & - & 0.027 \\
\hline NTM pulmonary disease & $2(4.3)$ & $0(0.0)$ & - & 0.227 \\
\hline Lung carcinoma & $2(4.3)$ & $3(6.0)$ & - & 1 \\
\hline ANCA associated systemtc vasculitis & $0(0.0)$ & $2(4.0)$ & - & 0.496 \\
\hline Diabetes & $9(19.6)$ & $8(16.0)$ & 0.209 & 0.648 \\
\hline Death & $2(4.3)$ & $0(0.0)$ & & 0.227 \\
\hline
\end{tabular}

Note: ${ }^{\mathrm{P}}$ value $<0.05$, the difference was statistically significant

Table 2: Comparison of microbiological examination results between the case group and the control group.

\begin{tabular}{|c|c|c|c|c|}
\hline Microbiological test results & Case group(\%) & Control group(\%) & Zvalue & P value \\
\hline Positive aspergillus in BALF culture & $21(45.70)$ & $0(0.00)$ & - & $0.001^{*}$ \\
\hline Positive aspergillus in BALF smear microscopy & $3(6.50)$ & $0(0.00)$ & - & 0.106 \\
\hline Serum GM value & $0.18(0.12-0.34)$ & $0.12(0.07-0.21)$ & -1.626 & 0.104 \\
\hline BALF GM value & $1.93(0.61-5.78)$ & $0.51(0.25-0.82)$ & -4.709 & $0.001^{*}$ \\
\hline
\end{tabular}

Note: ${ }^{*} \mathrm{P}$ value $<0.05$, the difference was statistically significant

Diagnostic effificacy of the BALF GM test. When the GM cutoff value was $0.5,0.8,0.9,1.0,1.2,1.5$, the sensitivity of BALF GM test decreased with the increase of GM cut-off value, and the specificity increased with the increase of GM cut-off value. When the diagnostic threshold of serum GM test was 0.5 and 1.0, the sensitivity decreased with the increase of the threshold, but the specificity did not change. BALF GM test had higher sensitivity but lower specificity than serum GM test (Table 3). The area under ROC curve of BALF-GM was 0.779(95\%CI: 0.684-0.874),standard error was $0.0487, \mathrm{Z}$ value was $5.727, \mathrm{P}=0.001$, Youden index was 0.4939 , when thre hold $>0.96$, the sensitivity and specificity were $67.4 \%, 82.0 \%$ respectively (Figure 1, Table 4 ). The area under ROC curve of serum-GM was 0.638(95\%CI: 0.439-0.807), standard error was $0.121, \mathrm{Z}$ value was $1.147, \mathrm{P}=0.255$, Youden index was 0.3116 , when threshold $>0.18$, Sen was $47.8 \%$, Spe was $83.3 \%$; When serum-GM threshold $\geq 0.18$, AUROC was the highest, for which the sensitivity and specificity were $45.5 \%, 83.3 \%$ respectively (Figure 2, Table 4).

Table 3: Diagnostic value of different GM test limits for BALF GM test.

\begin{tabular}{|c|c|c|c|c|c|}
\hline Cutoff value & Sen(\%) & Spe(\%) & PPV(\%) & NPV(\%) \\
\hline \multicolumn{7}{|c|}{ BALF } \\
\hline$\geq 0.5$ & 80.43 & 48 & 58.73 & 72.72 \\
\hline$\geq 0.8$ & 67.39 & 72 & 68.89 & 70.58 \\
\hline$\geq 0.9$ & 67.39 & 78 & 73.8 & 72.22 \\
\hline$\geq 1.0$ & 67.39 & 82 & 77.5 & 3.06 \\
\hline
\end{tabular}




\begin{tabular}{|l|l|l|l|l|l|}
\hline$\geq 1.2$ & 58.69 & 88 & 81.81 & 69.84 & 4.89 \\
\hline$\geq 1.5$ & 52.17 & 90 & 82.75 & \multicolumn{3}{|c|}{67.16} & 5.21 \\
\hline \multicolumn{7}{|c|}{ Serum } \\
\hline$\geq 0.5$ & 16.0 & 100 & 100 & 22.22 & - \\
\hline
\end{tabular}

Table 4: Comparison of ROC curve analysis parameters between BALF-GM test and serum GM test.

\begin{tabular}{|c|c|c|c|c|c|c|c|}
\hline & AUC(95\%CI) & Z-value & Youden index & Cutoff value & Sen(\%) & Spe(\%) & Pvalue \\
\hline BALF-GM & $0.779(0.684-0.874)$ & 5.72 & 0.4939 & 0.96 & 67.4 & 82 & $0.001^{*}$ \\
\hline Serum-GM & $0.638(0.439-0.807)$ & 1.147 & 0.3116 & 0.18 & 47.8 & 83.3 & 0.255 \\
\hline
\end{tabular}

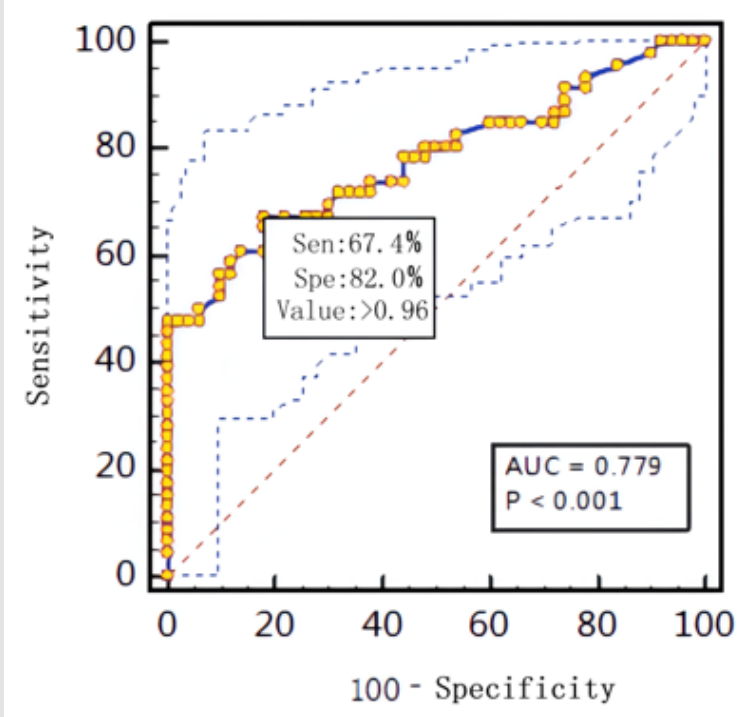

Figure 1: ROC curves of BALF-GM test in two groups.

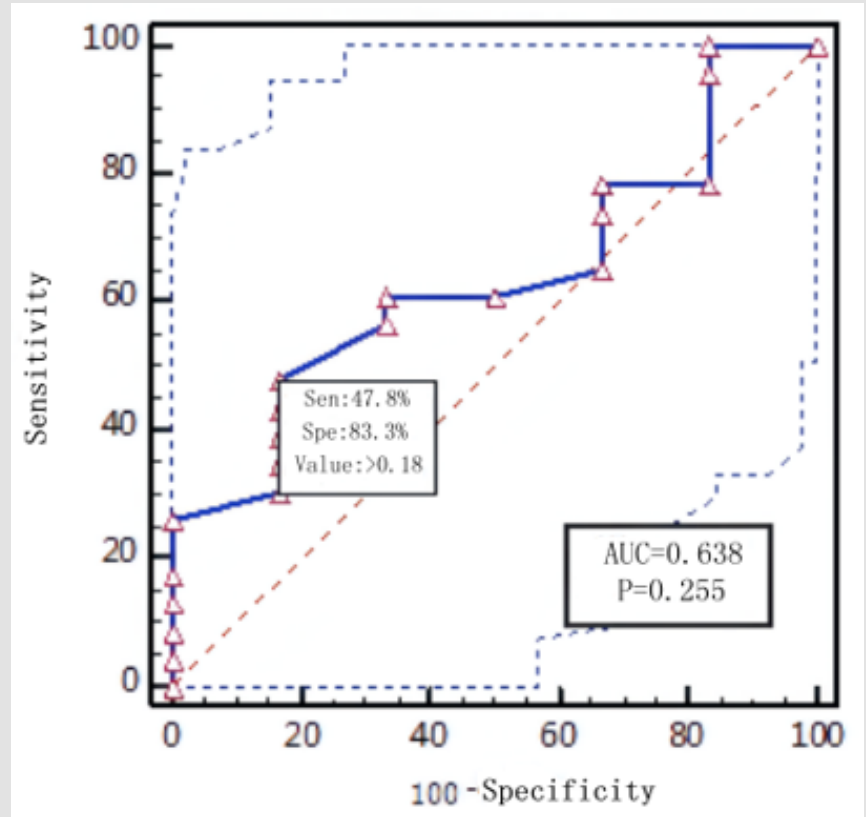

Figure 2: ROC curves of serum-GM test in two groups. 


\section{Discussion}

Structural lung disease is a major cause of pulmonary aspergillosis, including bronchiectasis, PTB, bronchial asthma, COPD, etc. Long-term and chronic diseases lead to the destruction of the normal anatomical and physiological structure of the lungs, the destruction of the mucosal barrier of respiratory epithelial cells, and increase the ability of aspergillosis to adhere to airway epithelium. In addition, cilium lodging and degeneration of airway epithelium and obstruction of clearance of respiratory secretion increase the chance of aspergillus infection [4,5]. This study also confirmed that patients with pulmonary aspergillosis had more chronic respiratory diseases in the case group than in the control group (bronchiectasis 58.7\% vs.12.0\%, P=0.001),PTB (82.6 vs. $20.0 \%, \mathrm{P}=0.001)$,COPD (43.5\% vs.22.0\%,P=0.025), which was consistent with the results reported in literature [6].The early clinical manifestations of pulmonary aspergillosis are not specific, and the typical chest CT findings are often related to the time of disease occurrence and the severity of lesion development, and the imaging findings cannot lead to a definite etiological diagnosis.

Traditional methods such as smear microscopy and fungal culture have long cycle, low positive rate and are susceptible to environmental pollution. Therefore, a variety of auxiliary examination methods are used to achieve the purpose of early diagnosis. Galactomannan (GM) is a specific polysaccharide of aspergillus cell wall. At present, GM can be detected clinically by blood, BALF, pleural effusion, cerebrospinal fluid and lung tissue, and it is one of the common antigens for the diagnosis of aspergillosis. A large number of existing studies have proved that the sen, spe, ppv and diagnostic coincidence rates of BALF were higher than those of serum GM. The results of this study showed that the cutoff values of BALF GM test were all higher than serum GM, which was consistent with the results of previous studies. The uniform diagnostic threshold of BALF GM has been disputed at home and abroad. The IDSA 2016 guidelines again recommended BALF GM and serum GM tests as laboratory tests for pulmonary aspergillosis. However, they did not specify a BALF GM value, but the diagnostic threshold of serum GM test was $\geq 0.5$ [2]. In 2019, EORTC/MSGERC scholars updated the definition of IFD, which clearly indicated for the first time that the clinical diagnostic threshold of BALF GM as pulmonary aspergillosis was: serum $\mathrm{GM} \geq 1.0,2$ BALF $\mathrm{GM} \geq 1.0$; or a single serum $\mathrm{GM} \geq 0.7+\mathrm{a}$ single $\mathrm{BALF} \mathrm{GM} \geq 0.8[7,8]$.

In this study, through ROC curve analysis, the AUROC of BALF GM test was 0.779(95\%CI:0.684-0.874). When BALF GM test limit> 0.96 ,Sen was $67.4 \%$,Spe was $78.0 \%$,PPV was $73.8 \%$, NPV was $72.2 \%$, PLR was 3.06.When serum GM limit was greater than 0.18,AUROC was the highest, Sen was $45.5 \%$,Spe was $83.3 \%$,and $\mathrm{P}=0.255$.The purpose of this study was to understand the value of BALF GM in the early diagnosis of pulmonary aspergillosis in patients with non- neutropenia complicated with pulmonary underlying diseases. Our results showed that the sensitivity of serum GM test was lower than BALF GM test regardless of setting GM $\geq 0.5, \geq 0.8$, or $\geq 1.0$ as the diagnostic threshold of BALF GM. When GM threshold was $\geq 0.5$, Sen,Spe,PPV of BALF GM were $80.43 \%, 48.0 \%, 58.73 \%$ respectively. When the BALF-GM threshold was increased to $\geq 1.0$, the PPV was significantly increased. Compared with previous studies $[9,10]$, BALF GM values of patients with chronic respiratory diseases were different from those of patients with traditional diseases such as neutropenia, hematological malignancies, parenchymal organ transplantation, hematopoietic stem cell transplantation, and immunosuppressant use.

At present, some scholars have proposed that different optimal diagnostic boundaries should be set for patients with different underlying diseases and different immune states, such as neutropenia and non-neutropenia [10], organ transplantation (including hematopoietic stem cell transplantation) and non-solid organ transplantation $[11,12]$, hematological malignancies $[13,14]$, etc. Similarly, the interpretation of BALF GM test results should also be based on the full assessment of the underlying diseases and immune status of patients to determine the optimal BALF GM diagnostic threshold for various patients, so as to improve the diagnostic efficacy of BALF GM in the diagnosis of pulmonary aspergillosis in different populations. Research and clinical practice at home and abroad have found that many factors affecting GM tests cause false positives and false negatives in GM tests, which often confuses clinical work and even leads to misdiagnosis, missed diagnosis and excessive antifungal treatment.

In this study, it was found that BALF GM value was higher in some patients without aspergillus infection in the control group, while BALF GM value was lower in a small number of patients with aspergillus infection in the case group, resulting in false negative in addition to sample dilution during BALF collection, which might also be related to the use of antifungal drugs. A recent review suggested that false negatives in GM tests were associated with the use of antifungal active agents and myxolytic agents [15]. Using beta lactam classes of antibiotics (especially piperacillin/ he azole temple, amoxicillin/clavulanic acid potassium, etc.), intravenous use of parenteral nutrition, blood product containers containing glucose acid, severe gastrointestinal mucous membrane inflammation, multiple myeloma will lead to GM false positive [15]. Clinical cases have also reported that contamination of sterile containers could lead to false positives of GM [16]. According to previous studies and the results of this study, the early diagnosis of pulmonary aspergillosis requires combining imaging examination, histopathology, smear microscopy, fungal culture, aspergillosis antigen detection, aspergillosis antibody detection, and molecular biological examination. 


\section{Conclusion}

In this study, BALF GM test is more valuable than serum GM test for diagnosis. BALF GM test is more significant for the diagnosis of pulmonary aspergillosis. The best limit, sensitivity and specificity of BALF GM test are $0.96,67.4 \%$ and $82.0 \%(\mathrm{P}=0.01)$. The optimal threshold of BALF GM may vary with host-based diseases and even with different species of Aspergillus. BALF GM value of pulmonary aspergillosis under different immune states needs more clinical data. At the same time, when serum GM and BALF GM are used in clinical practice, it is necessary to fully understand and identify the false positive and false negative of GM, and to diagnose pulmonary aspergillosis by integrating patient factors, clinical manifestations, imaging examination and pathogenic microbial examination.

\section{Acknowledgement}

I should express my gratitude to Dr. Dexian Li in the Guangzhou Thoracic Hospital, Dr. Jiquan Guo in the Guangdong Provincial People's Hospital, and Dr. Huai Liao in the First Affiliated Hospital of Sun Yat-sen University for their assistance to collect the BALF specimens.

\section{References}

1. (2020) GBD Chronic Respiratory Disease Collaborators. Prevalence and attributable health burden of chronic respiratory diseases, 1990-2017: a systematic analysis for the Global Burden of Disease Study 2017. Lancet Respir Med 8(6): 585-596.

2. Patterson TF, Thompson GR, Denning DW, Fishman JA, Hadley S, et al. (2016) Practice Guidelines for the Diagnosis and Management of Aspergillosis: 2016 Update by the Infectious Diseases Society of America. Clin Infect Dis 63(4): e1-60.

3. Denning DW, Cadranel J, Beigelman-Aubry C, Ader F, Chakrabarti A, et al. (2016) Chronic pulmonary aspergillosis: rationale and clinical guidelines for diagnosis and management. Eur Respir J 47(1): 45-68.

4. Latgé JP, Chamilos G (2019) Aspergillus fumigatus and Aspergillosis in 2019. Clin Microbiol Rev 33(1): e00140-e00148.

5. Denning DW, Pleuvry A, Cole DC (2011) Global burden of chronic pulmonary aspergillosis as a sequel to pulmonary tuberculosis. Bull World Health Organ 89(12): 864-872.

\section{ISSN: 2574-1241}

DOI: 10.26717/BJSTR.2021.39.006312

Chang-ran Zhang. Biomed J Sci \& Tech Res

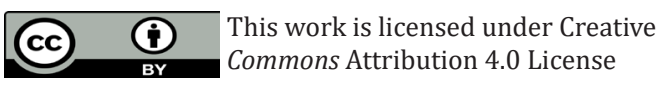

Submission Link: https://biomedres.us/submit-manuscript.php
6. Kanj A, Abdallah N, Soubani AO (2018) The spectrum of pulmonary aspergillosis. Respir Med141: 121-31.

7. Donnelly JP, Chen SC, Kauffman CA, Steinbach WJ, Baddley JW, et al. (2020) Revision and Update of the Consensus Definitions of Invasive Fungal Disease From the European Organization for Research and Treatment of Cancer and the Mycoses Study Group Education and Research Consortium. Clin Infect Dis 71(6): 1367-1376.

8. Mercier T, Castagnola E, Marr KA, Wheat LJ, Verweij PE, et al. (2021) Defining Galactomannan Positivity in the Updated EORTC/MSGERC Consensus Definitions of Invasive Fungal Diseases. Clin Infect Dis 72(2): S89-94.

9. Zhang XB, Chen GP, Lin QC, Lin X, Zhang HY, et al. (2013) Bronchoalveolar lavage fluid galactomannan detection for diagnosis of invasive pulmonary aspergillosis in chronic obstructive pulmonary disease. Med Mycol 51(7): 688-695.

10. De Heer K, Gerritsen MG, Visser CE, Leeflang MM (2019) Galactomannan detection in broncho-alveolar lavage fluid for invasive aspergillosis in immunocompromised patients. Cochrane Database Syst Rev 5(5): D12399.

11. De Mol M, De Jongste JC, Van Westreenen M, Merkus PJ, De Vries AH, et al. (2013) Diagnosis of invasive pulmonary aspergillosis in children with bronchoalveolar lavage galactomannan. Pediatr Pulmonol 48(8): 789-796.

12. Husain S, Bhaskaran A, Rotstein C, Li Y, Bhimji A, et al. (2018) A strategy for prevention of fungal infections in lung transplantation: Role of bronchoalveolar lavage fluid galactomannan and fungal culture. J Heart Lung Transplant 37(7): 886-894.

13. Gupta A, Capoor MR, Shende T, Sharma B, Mohindra R, et al. (2017) Comparative evaluation of galactomannan test with bronchoalveolar lavage and serum for the diagnosis of invasive aspergillosis in patients with hematological malignancies. J Lab Physicians 9(4): 234-238.

14. Heldt S, Prattes J, Eigl S, Spiess B, Flick H, et al. (2018) Diagnosis of invasive aspergillosis in hematological malignancy patients: Performance of cytokines, Asp LFD, and Aspergillus PCR in same day blood and bronchoalveolar lavage samples. J Infect 77(3): 235-241.

15. Paiva JA, Pereira JM (2019) Biomarkers of fungal lung infection. Curr Opin Infect Dis 32(2): 136-142.

16. Kathar SS, Mullerpattan J, Shetty A, Udwadia ZF (2017) Bronchoalveolar lavage contamination by sterile containers: A unique and unrecognized cause of a false-positive galactomannan. Lung India 34(6): 572-573.

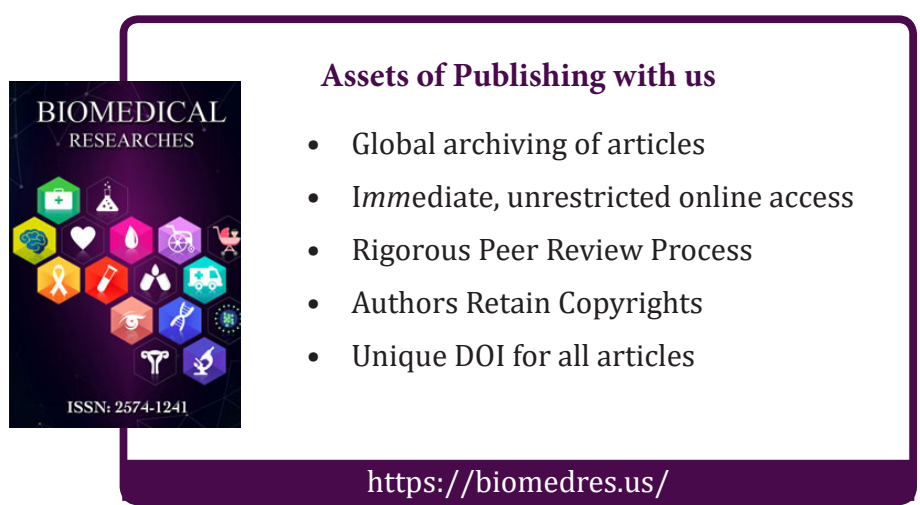

